\title{
Cinnamon Attenuates Neonatal Streptozotocin (nSTZ) Induced Pre-diabetic Renal Alterations in the SD Rat Model via Maintenance of Proper Glucose Homeostasis and Antioxidant Property
}

\author{
K. Siva Kesavarao ${ }^{1}$, T. Raghavarao ${ }^{2}$ and P. Suryanarayana ${ }^{1}$ * \\ ${ }^{1}$ Lipid chemistry Division, National Institute of Nutrition, Hyderabad, Telangana, India \\ ${ }^{2}$ Andhra university, Biochemistry Division, Visakhapatnam, India \\ *Corresponding author
}

Keywords

Pre-diabetes,

Diabetic

nephropathy,

Cinnamon, STZ

Article Info

Accepted:

10 December 2018

Available Online:

10 January 2019

\section{A B S T R A C T}

The major purpose of our study is to evaluate the impact of cinnamon on long term prediabetic induced renal alterations caused by intraperitonial induction of neonatal streptozotocin (nSTZ). Two-day old male Sprague Dawley $(\mathrm{SD})$ rat pups $(\mathrm{n}=30)$ received a single intra peritoneal injection of STZ ( $90 \mathrm{mg} / \mathrm{kg} \mathrm{bw}$ ) dissolved in $0.1 \mathrm{M}$ citrate buffer, $\mathrm{pH}$ 4.5 while Control pups $(\mathrm{n}=8)$ received the vehicle alone. All rats were maintained on an AIN-93G/M diet in individual cages and a subset of pre-diabetic animals received 3\% cinnamon in the AIN-93 diet. The majority of nSTZ rats exhibited impaired glucose tolerance (IGT) $(2 \mathrm{~h}$ glucose $>140 \mathrm{mg} / \mathrm{dl})$ or pre-diabetes by 2 months and the same was maintained up to the 10 months as evidenced by OGTT as well as blood glucose levels. Functional abnormalities of the kidney were studied by plasma as well as urinary parameters. Renal pathological changes were observed by H\&E staining and immunoblotting was performed to determine the protein expressions of nephrin and podocin. Urinary albumin, urea levels are elevated, whereas plasma albumin levels are decreased in pre-diabetes rats compared to control rats. Protein and mRNA expression levels of nephrin and podocin were lower in PD animals when compared to control. Cinnamon cinnamon $(\mathrm{CN})$ reduces urinary albumin levels, and also marginally prevented the loss of protein and mRNA expressions of nephrin and podocin. Our findings might provide a basis for long term pre-diabetes induced renal alterations ameliorated by cinnamon, but the exact molecular mechanisms still require further elucidation.

\section{Introduction}

Diabetic nephropathy (DN) is slow and progressive loss of kidney function over a period of several years is another important microvascular complication of diabetes and is defined as glomerular filtration rate $<60$ $\mathrm{mL} / \mathrm{min} / 1.73 \mathrm{~m}^{2}$ for 3 months or more (Levey and Mulrow, 2005). Diabetic nephropathy is the primary cause of chronic kidney disease (CKD) which is leading cause of renal failure, also known as end-stage renal disease (ESRD) (Fong et al., 2004; Mogensen, 1999; Ruggenenti and Remuzzi, 1998), which impair the quality of life and also increases morbidity and mortality. In addition, treatment of CKD 
is involved with high cost throughout the treatment period, especially when the person enters to ESRD stage (Agarwal 2005; Ahlawat et al., 2017). This leads to an increasing financial burden on both individuals as well as on health care systems.

The incidence/prevalence and progression of DN may depend upon the type of diabetes, severity of hyperglycemia and duration of diabetes (Shahbazian and Rezaii, 2013; Unnikrishnan et al., 2007). Around 20-30\% of both type- 1 and type- 2 diabetic subjects have an evidence of diabetic nephropathy (Shahbazian and Rezaii, 2013) and nearly $30 \%$ of chronic renal failures are due to diabetic nephropathy in India (Agarwal and Dash, 2000). According to the earlier Indian studies, the prevalence of diabetic nephropathy varied from $2.2 \%$ to $31 \%$ ). The progression of diabetic nephropathy to ESRD is involved with several progressive steps which includes hyperfiltration, microalbuminuria (early nephropathy), macroalbuminuria with decreased glomerular filtration rate (GFR) (Palatini, 2012) followed by the structural alterations like glomerular basement membrane thickening, and glomerular sclerosis (Pourghasem et al., 2015).

Cinnamon $(\mathrm{CN})$ is derived from the inner bark of Cinnamomum trees and has a long history as a culinary and medicinal plant. There are two primary varieties of $\mathrm{CN}$, including Cinnamomum cassia and Cinnamomum zeylanicum. Cinnamomum zeylanicum is known as the 'true cinnamon'; it is lighter in colour, sweeter and has a more delicate flavour than Cinnamomum cassia, which has a stronger taste and is darker. Short-term ingestion of cinnamon on in vivo glucose tolerance in healthy human volunteers has also shown fasting glucose and two hour glucose lowering effect (Solomon and Blannin, 2007). In another study, Cinnamon ingestion reduced the glucose response to OGTT in healthy humans (Solomon and Blannin, 2009). In addition, it also lowers the postprandial glucose in healthy subjects (Hlebowicz et al., 2007).

In addition to its hypoglycemic potentials $\mathrm{CN}$ has also shown its beneficial effects in delay of prevention of various complications. Earlier our group demonstrated beneficial effects of procyanidin-B2 enriched fraction of $\mathrm{CN}$ in type-1 diabetes induced cataract and nephropathy in rat model (Muthenna et al., 2013; Muthenna et al., 2014). These beneficial effects of procyanidin-B2 enriched $\mathrm{CN}$ fraction is through its antiglycating potentials. The other group has shown beneficial effect of cinnamon oil on early stage diabetic nephropathy against alloxan-induced renal damage and its effect mainly through its antioxidant and antidiabetic properties (Mishra et al., 2010).

However, beneficial effects of cinnamon in pre-diabetes induced renal complications are not well studied. Hence the main purpose of this study is to assess the beneficial effects of $\mathrm{CN}$ in vivo against longterm pre-diabetes induced renal abnormalities using $\mathrm{nSTZ}$ rat model.

\section{Results and Discussion}

\section{Effect of $\mathrm{CN}$ on plasma glucose and insulin response during OGTT}

Glucose response during OGTT two months after STZ injection showed significant higher plasma glucose levels at all time points, except 0 min, in untreated PD rats compared to those of control rats indicating development of IGT but not IFG (Fig. 1). Insulin levels were significantly lower at $30 \mathrm{~min}$ after glucose challenge (Fig. 1C) in untreated PD rats when compared to control rats, but insulin levels at 0 and 120 min were similar between control 
and untreated PD rats. In addition, there was no difference in HOMA-IR index between untreated PD and control rats indicating that nSTZ animals did not develop insulin resistance. These findings are well correlated with the previous experiment reported in chaper-2 and also with our previous study (Patil et al., 2014).

In addition to these observations, OGTT graphs before termination or at ten months of the experiment showed significant higher plasma glucose levels at all time points in untreated PD rats, compared to those of control rats indicating these animals maintained IGT and also developed IFG (Fig. $1)$. Whereas, insulin levels were significantly lower at 30, $60 \mathrm{~min}$ after glucose challenge (Fig. 1D) in untreated PD rats when compared to control rats, but there is no difference in insulin levels at 0 and $120 \mathrm{~min}$ between control and untreated PD rats (Fig. 1D). Together these OGTT glucose and insulin responses indicated that $\mathrm{nSTZ}$ rats developed of IGT by two months and its persistence for at least 10 months. Moreover, CN-treated PD rats exhibited significant hypoglycemic as the 2 hours glucose completely normalised and it also improved insulin levels at $30 \mathrm{~min}$ and 120 min when compared to untreated PD rats (Fig. $1)$.

\section{Clinical nephropathy parameters}

Clinical parameters related to nephropathy were estimated in urine and presented in Table 1. Urinary albumin, creatinine levels were significantly higher and urinary urea levels were marginally higher in untreated PD rats than control rats indicating initiation of renal abnormalities in PD rats. Interestingly, urinary creatinine significantly lower and albumin, urea and urine output were slightly low in the $\mathrm{CN}$ treated PD rats than in untreated PD rats indicating its protective role in PD induced renal abnormalities (Table 1). Similarly, Plasma parameters related to nephropathy were estimated and presented in Table 2. Plasma urea, blood urea nitrogen (BUN) and glomerular filtration rate (GFR) were significantly higher in untreated PD rats when compared to control rats. However, plasma albumin significantly lower and creatinine levels were marginally low in untreated PD rats when compared the control rats indicating altered renal function in PD rats. Feeding of $\mathrm{CN}$ to $\mathrm{PD}$ rats has prevented renal abnormalities by preventing altered albumin, creatinine, urea, BUN and GFR (Table 2).

\section{Kidney morphology by Haematoxylin and Eosin (H\&E)}

In addition to the clinical parameters, we have also studied morphology of kidney in control, $\mathrm{PD}$ and $\mathrm{PD}+\mathrm{CN}$ group rats by $\mathrm{H} \& \mathrm{E}$. As observed in the previous experiment, in this study also there was a modest glomerular lesion in untreated PD rat kidney sections when compared to control rat kidney sections indicating development of structural alterations in PD state. Feeding of $\mathrm{CN}$ to PD rats prevent glomerular lesions compared to untreated PD rats (Fig. 2). These protective changes were similar as reported by the other studies in alloxan or STZ induced kidney damages or nephropathy in rats (Mishra et al., 2010; Qusti et al., 2016)

\section{Nephrin and podocin mRNA Expression in kidney by qRT-PCR}

There was a marginal down regulation of nephrin mRNA expression and significant down regulation of podocin mRNA expression was observed in untreated PD group compared to the control group (Fig. 3) indicates the development of nephropathy in the PD rats. This data further supporting the clinical and histological results. Feeding of $\mathrm{CN}$ to PD rats significantly prevented down regulation of nephrin and podocin mRNA expressions compared to the PD rats (Fig. 3). 
Nephrin and podocin protein expressions by Western blot

To further substantiate our qPCR results, we have also used western blotting method to study the expression of nephrin and podocin at protein levels in the control, $\mathrm{PD}$ and $\mathrm{CN}$ treated PD rat kidneys.

As expected, the relative expression of nephrin and podocin was slightly lower in untreated PD rat kidneys than in the control rat kidneys indicating development of renal abnormalities. Feeding of $\mathrm{CN}$ to $\mathrm{PD}$ rats marginally prevented loss of nephrin, but not podocin protein when compared to untreated PD rat kidneys (Fig. 4).
Oxidative stress and antioxidant enzyme in the kidney

MDA levels were similar in all three groups, but significantly enhanced the activity of SOD in the untreated PD group as compared to that of control group which indicates that longterm pre-diabetes also increases oxidative stress (Table 3). CN-treated PD rats showed SOD activity near to the controls when compared to untreated PD rats, indicating that $\mathrm{CN}$ prevented oxidative stress in the PD rat kidney due to its antioxidant property (Table 3). Similar findings were reported in alloxan or STZ induced oxidative stress in kidney in rats (Mishra et al., 2010; Qusti et al., 2016).

Table.1 Urinary nephropathy parameters

\begin{tabular}{|c|c|c|c|}
\hline \multirow[t]{2}{*}{ Parameters } & \multicolumn{3}{|c|}{ Groups } \\
\hline & Control & PD & $\mathbf{P D}+\mathbf{C N}$ \\
\hline Albumin (mg/24 h) & $20.73 \pm 1.26$ & $42.46 \pm 4.90 *$ & $33.06 \pm 3.10$ \\
\hline Creatinine (mg/24 h) & $12.43 \pm 1.16$ & $17.33 \pm 1.65^{*}$ & $11.56 \pm 1.08^{\$}$ \\
\hline Urea (mg/24 h) & $610 \pm 53$ & $890 \pm 111$ & $690 \pm 61$ \\
\hline Water intake (mL/24 h) & $33.13 \pm 0.91$ & $35.80 \pm 1.42$ & $40.16 \pm 2.98$ \\
\hline Urine output (mL/24 h) & $8.40 \pm 1.20$ & $13.60 \pm 1.72$ & $10.84 \pm 1.62$ \\
\hline
\end{tabular}

Table.2 Plasma nephropathy parameters

\begin{tabular}{|c|c|c|c|}
\hline \multirow{2}{*}{ Parameters } & \multicolumn{3}{|c|}{ Groups } \\
\hline & Control & PD & $\mathbf{P D}+\mathbf{C N}$ \\
\hline Albumin (mg/dL) & $24.51 \pm 2.32$ & $22.56 \pm 0.52 *$ & $24.37 \pm 0.95$ \\
\hline Creatinine (mg/dL) & $0.65 \pm 0.03$ & $0.63 \pm 0.03$ & $0.64 \pm 0.02$ \\
\hline Urea $(\mathrm{mg} / \mathrm{dL})$ & $25.69 \pm 1.59$ & $33.32 \pm 2.35^{*}$ & $34.56 \pm 1.83$ \\
\hline BUN (mg/dL) & $11.97 \pm 0.74$ & $15.52 \pm 1.09 *$ & $16.11 \pm 0.85$ \\
\hline GFR/kg/bw & $3.20 \pm 0.09$ & $4.82 \pm 0.39 *$ & $3.12 \pm 0.41^{\$}$ \\
\hline
\end{tabular}


Table.3 Malondialdehyde (MDA) and superoxide dismutase (SOD) activity in control, PD and $\mathrm{PD}+\mathrm{CN}$ group rat kidneys

\begin{tabular}{|c|c|c|c|}
\hline \multirow[t]{2}{*}{ Parameters } & \multicolumn{3}{|c|}{ Groups } \\
\hline & Control & PD & $\mathbf{P D}+\mathbf{C N}$ \\
\hline MDA (nmol/g kidney) & $0.62 \pm 0.03$ & $0.60 \pm 0.04$ & $0.59 \pm 0.07$ \\
\hline SOD (U/min/100 mg protein) & $32.48 \pm 1.53$ & 43.01 $\pm 2.11 *$ & $34.21 \pm 2.26 \$$ \\
\hline \multicolumn{4}{|c|}{$\begin{array}{l}\text { Lipid peroxidation [malondialdehyde (MDA)] was expressed as } \mathrm{nmol} / \mathrm{g} \text { kidney } \\
\text { and superoxide dismutase (SOD, enzyme) was expressed as Units/min } / 100 \mathrm{mg} \\
\text { proteins. Values are mean } \pm \mathrm{SE} \text {, } \mathrm{n}=6-9 \text { animals per group. }{ }^{*}<<0.05 \mathrm{vs} \text {. Control } \\
\text { and } \$ \mathrm{p}<0.05 \text { vs. PD. }<<0.05 \text { was considered as statistically significant by ANOVA }\end{array}$} \\
\hline
\end{tabular}

Fig.1 Glucose (A and B) and insulin (C and D) responses at various time points $(0,30,60$ and $120 \mathrm{~min}$ ) during OGTT on overnight-fasted rats. OGTT was conducted in control, PD and $\mathrm{PD}+\mathrm{CN}$ group rats after $2^{\text {nd }}$ and $10^{\text {th }}$ month of STZ injection. Values are mean $\pm S E, n=9-12$ animals per group. ${ }^{*} \mathrm{p}<0.05$ vs. Control; $\$ \mathrm{p}<0.05$ vs. PD group. $\mathrm{p}<0.05$ was considered as statistically significant by independent-test and ANOVA. OGTT, Oral glucose tolerance test

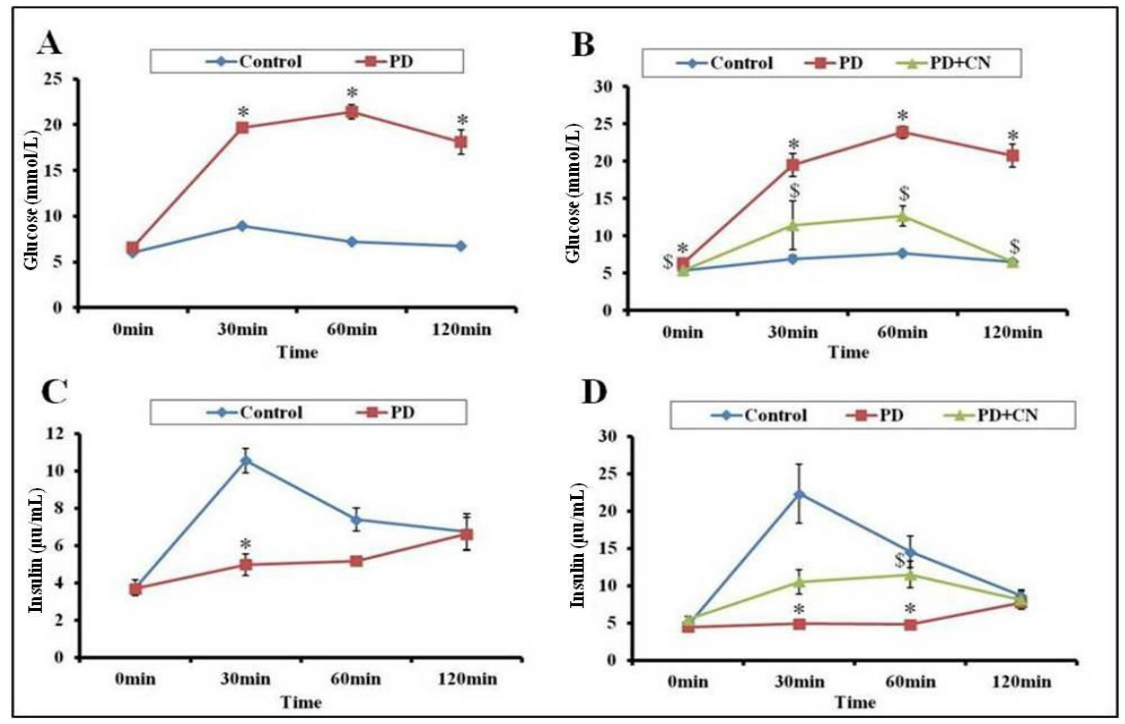

Fig.2 Representative kidney sections of control, PD and PD+CN groups stained with haematoxylin and eosin $(\mathrm{H} \& \mathrm{E})$. The stained kidney sections were examined under a light microscope and images were captured at a magnification of $400 \mathrm{x}$

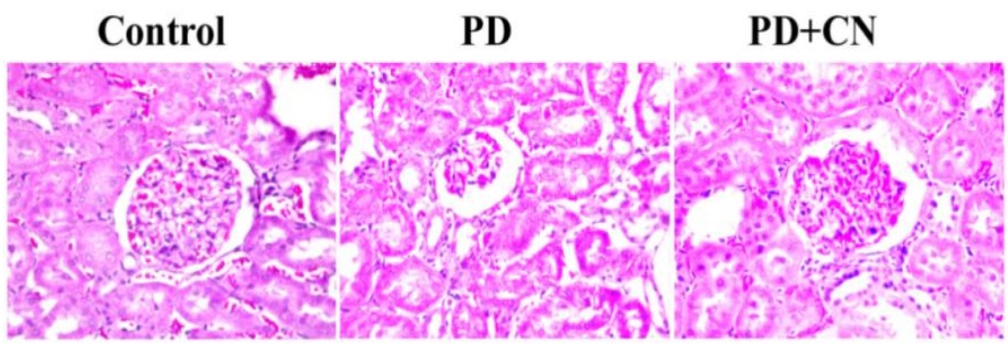


Fig.3 Nephrin and podocin mRNA expression in rat kidneys from the control, PD and PD+CN groups by qRT-PCR. Values are mean \pm SE of three independent $m R N A$ preparations for each group. ${ }^{*} \mathrm{p}<0.05$ vs. Control; $\$ \mathrm{p}<0.05$ vs. PD. $\mathrm{p}<0.05$ was considered as statistically significant by ANOVA

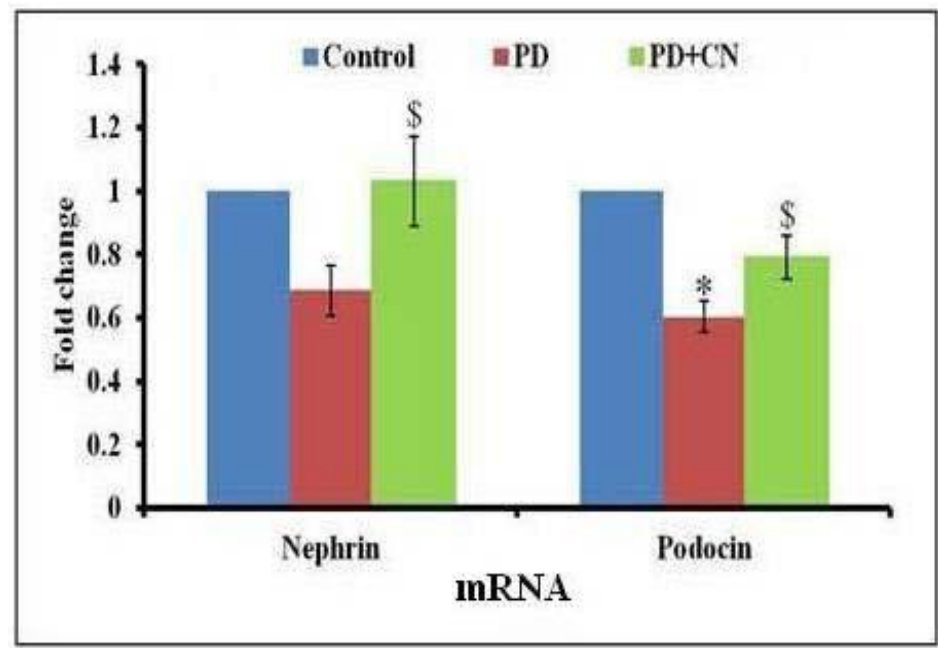

Fig.4 Representative western blot (A) and densiometry (B) of nephrin and podocin protein expression in the kidney of control, $\mathrm{PD}$ and $\mathrm{PD}+\mathrm{CN}$ group rats. Equal protein loading was confirmed with a $\beta$-actin antibody. The graphs represent comparative densities of each band normalised to the corresponding level of $\beta$-actin. Values are mean \pm SE of three independent protein preparations for each group

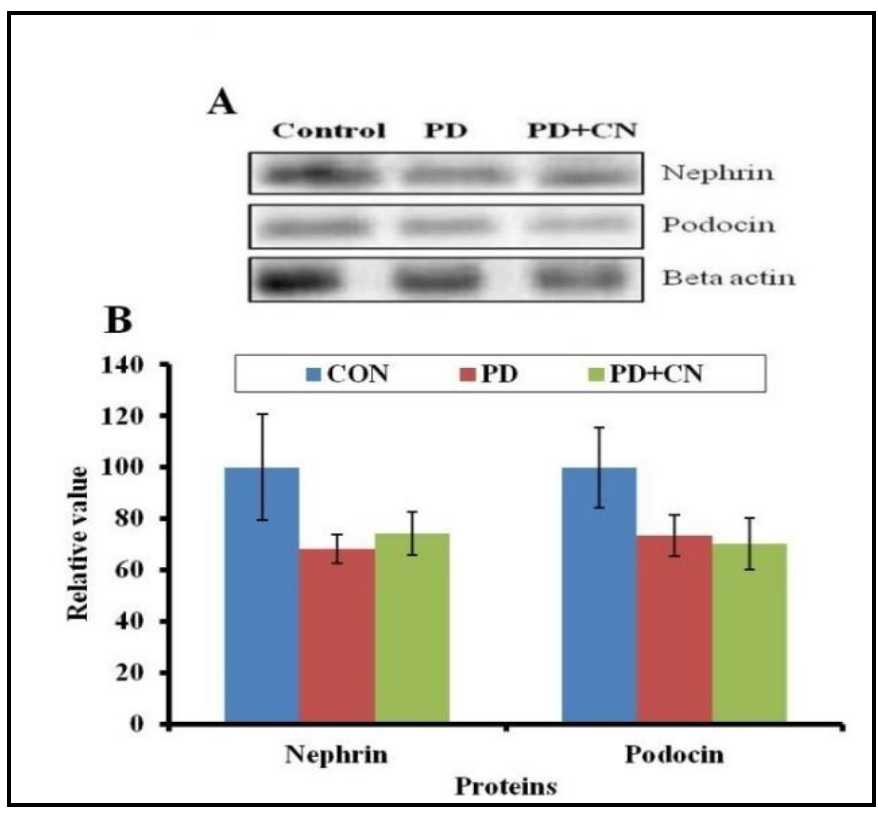

In conclusion, our findings might provide a basis for long term pre-diabetes induced renal alterations attenuated by cinnamon due to its hypoglycemic and antioxidant property, but the exact molecular mechanisms still require further elucidation. 


\section{Acknowledgements}

K.S.K. Rao received a research fellowship from the Indian Council of Medical Research, Government of India.

\section{Funding sources}

P.S.N. Received grants from the Department of Biotechnology, Government of India (Grant No: BT/PR3446/BRB/10/969/2011) and National Institute of Nutrition (Indian Council of Medical research, Government of India) for intramural funding (\#12-BS11).

Conflict of Interest: None declared.

\section{References}

Acharya, V.N. and K.P. Chawla (1978) Diabetic nephropathy--a review. J Postgrad Med, 24(3): 138-146.

Agarwal, S.K (2005) Chronic kidney disease and its prevention in India. Kidney Int Suppl, 98: 41-45.

Agarwal, S.K. and S.C. Dash (2000) Spectrum of renal diseases in Indian adults. J Assoc Physicians India, 48(6): 594-600.

Ahlawat, R., P. Tiwari and S. D'Cruz (2017) Direct Cost for Treating Chronic Kidney Disease at an Outpatient Setting of a Tertiary Hospital: Evidence from a Cross-Sectional Study. Value Health Reg Issues, 12: 36-40.

Chugh, K.S., V. Sakhuja, H.S. Malhotra and B.J. Pereira (1989) Changing trends in acute renal failure in third-world countries--Chandigarh study. Q J Med, 73(272): 1117-1123.

Fong, D.S., L.P. Aiello, F.L. Ferris and R. Klein (2004) Diabetic retinopathy. Diabetes Care, 27(10): 2540-2553.

Hlebowicz, J., G. Darwiche and O. Björgell, L.O. Almér (2007) Effect of cinnamon on postprandial blood glucose, gastric emptying, and satiety in healthy subjects. Am J Clin Nutr, 85(6): 15521556.

John, L., P.S. and Rao A.S. (1991) Prevalence of diabetic nephropathy in non-insulin dependent diabetics. Indian $\mathbf{J}$ Med Res, 94: 24-29.

Levey, A.S. and C.D. Mulrow (2005) An editorial update: what level of blood pressure control in chronic kidney disease? Ann Intern Med, 143(1): 7981.

Mishra, A., R. Bhatti, A. Singh and M.P Singh Ishar (2010) Ameliorative effect of the cinnamon oil from Cinnamomum zeylanicum upon early stage diabetic nephropathy. Planta Med, 76(5): 412-417.

Mogensen, C.E (1999) Microalbuminuria, blood pressure and diabetic renal disease: origin and development of ideas. Diabetologia, 42(3): 263-285.

Muthenna, P., G. Raghu, C. Akileshwari, S.N. Sinha, P. Suryanarayana and G.B. Reddy (2013) Inhibition of protein glycation by procyanidin-B2 enriched fraction of cinnamon: delay of diabetic cataract in rats. IUBMB Life, 65(11): 941-950.

Muthenna, P., G. Raghu, P.A. Kumar, M.V. Surekha and G.B. Reddy (2014) Effect of cinnamon and its procyanidinB2 enriched fraction on diabetic nephropathy in rats. Chem Biol Interact, 222: 68-76.

Palatini, P (2012) Glomerular hyperfiltration: a marker of early renal damage in prediabetes and pre-hypertension. Nephrol Dial Transplant, 27(5): 1708-1714.

Patil, M.A., P. Suryanarayana, P. Uday Kumar, M. Srinivas and G.B. Reddy (2014) Evaluation of Neonatal Streptozotocin Induced Diabetic Rat Model for the Development of Cataract. Oxidative Medicine and Cellular Longevity, 2014: 1-10. 
Pourghasem, M., H. Shafi and Z. Babazadeh (2015) Histological changes of kidney in diabetic nephropathy. Caspian $\mathrm{J}$ Intern Med, 6(3): 120-127.

Qusti, S., H.A. Rabey and S.A. Balashram (2016) The Hypoglycemic and Antioxidant Activity of Cress Seed and Cinnamon on Streptozotocin Induced Diabetes in Male Rats. Evid Based Complement Alternat Med, 2016: 1-15. Rajapurkar, M.M., G.T. John, A.L. Kirpalani, G. Abraham, S.K. Agarwal, A.F. Almeida, S. Gang, A. Gupta, G. Modi, D. Pahari, R. Pisharody, J. Prakash, A. Raman, D.S. Rana, R.K. Sharma, R.N. Sahoo, V. Sakhuja, R.R. Tatapudi and V. Jha (2012) What do we know about chronic kidney disease in India: first report of the Indian CKD registry. BMC Nephrol, 13: 1-8.

Ruggenenti, P. and G. Remuzzi (1998) Nephropathy of type-2 diabetes mellitus. J Am Soc Nephrol, 9: 2157-
2169.

Shahbazian, H. and I. Rezaii (2013) Diabetic kidney disease; review of the current knowledge. J Renal Inj Prev, 2(2): 7380.

Solomon, T.P and A.K. Blannin (2007) Effects of short-term cinnamon ingestion on in vivo glucose tolerance. Diabetes Obes Metab, 9(6): 895-901.

Solomon, T.P and A.K. Blannin (2009) Changes in glucose tolerance and insulin sensitivity following 2 weeks of daily cinnamon ingestion in healthy humans. Eur J Appl Physiol, 105(6): 969-976.

Unnikrishnan, R.I., M. Rema, R. Pradeepa, M. Deepa, C.S. Shanthirani, R. Deepa and V. Mohan (2007) Prevalence and risk factors of diabetic nephropathy in an urban South Indian population: the Chennai Urban Rural Epidemiology Study (CURES 45). Diabetes Care, 30(8): 2019-2024.

\section{How to cite this article:}

Siva Kesavarao, K., T. Raghavarao and Suryanarayana, P. 2019. Cinnamon Attenuates Neonatal Streptozotocin (nSTZ) Induced Pre-diabetic Renal Alterations in the SD Rat Model via Maintenance of Proper Glucose Homeostasis and Antioxidant Property. Int.J.Curr.Microbiol.App.Sci. 8(01): 1047-1054. doi: https://doi.org/10.20546/ijcmas.2019.801.114 